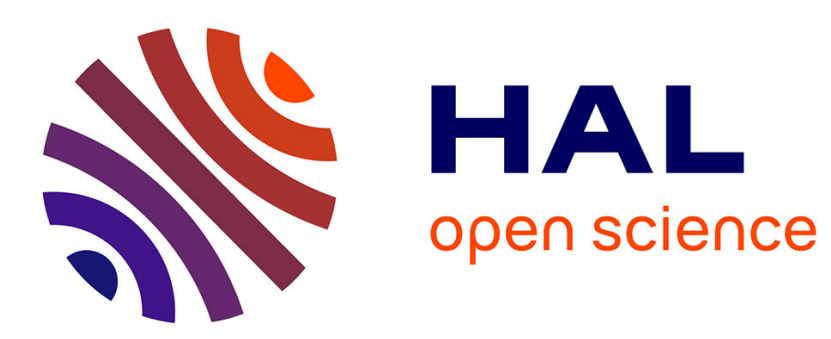

\title{
L'institutionnalisation des désaccords éthiques : le cas de la réanimation néonatale
}

\author{
Caroline Guibet Lafaye
}

\section{To cite this version:}

Caroline Guibet Lafaye. L'institutionnalisation des désaccords éthiques: le cas de la réanimation néonatale. Revue de Métaphysique et de Morale, 2010, 3, p. hal-00358927

\section{HAL Id: hal-00358927 \\ https://hal.science/hal-00358927}

Submitted on 4 Feb 2009

HAL is a multi-disciplinary open access archive for the deposit and dissemination of scientific research documents, whether they are published or not. The documents may come from teaching and research institutions in France or abroad, or from public or private research centers.
L'archive ouverte pluridisciplinaire HAL, est destinée au dépôt et à la diffusion de documents scientifiques de niveau recherche, publiés ou non, émanant des établissements d'enseignement et de recherche français ou étrangers, des laboratoires publics ou privés. 


\title{
L'institutionnalisation des désaccords éthiques : \\ le cas de la réanimation néonatale
}

\author{
Caroline Guibet LAfaye \\ Centre Maurice Halbwachs \\ CNRS \\ 48, bd Jourdan, F-75014 Paris (France) \\ caroline.guibet-lafaye@ens.fr
}

Résumé: La réanimation néonatale donne lieu à une demande publique et institutionnelle d'élaboration de normes, appelée par les dilemmes éthiques, induits par la pratique d'arrêt de vie ou de non réanimation. Ces dilemmes sont pratiquement levés par une référence aux « grands principes » qui régissent généralement la pratique médicale (dignité humaine, droit à la vie, droit à l'autonomie, obligation de soins, principe de non nuisance) ainsi que par l'exigence de suivre, pour procéder aux arrêts de vie, une « démarche transparente et progressive », incluant une dimension délibérative.

Dans la mesure où, en dépit de ces conditions, des désaccords éthiques légitimes demeurent et s'avèrent à l'analyse insolubles, il paraît nécessaire d'institutionnaliser ces désaccords afin de permettre aux personnes de "vivre ensemble» et de «travailler ensemble», en dépit de leurs divergences éthiques, toutes fondées sur de « bonnes raisons » morales.

Le service de réanimation néonatale est un lieu où la mort « décidée » est un phénomène fortement répandu puisque un décès sur deux fait suite à une décision d'arrêt de réanimation ${ }^{1}$. Pourtant, dans cet espace la technologie est plus aboutie que le code de la loi ou que les recommandations éthiques concernant ces pratiques. Les enfants qui survivent, après être nés en situation de détresse vitale et réanimés, sont fréquemment atteints de lésions cérébrales sévères responsables de séquelles neurologiques qui nécessiteront des soins lourds toute leur $v i^{2}$. Près de la moitié des handicaps moteurs sévères de l'enfance sont associés à ces réanimations. Lorsqu'ils sont interrogés, les néonatologistes français, à une majorité significative, considèrent que des gestes dont le but avéré est de mettre un terme à la vie du nouveau-né dans des situations qu'ils jugent particulièrement «insupportables » ou désespérées, sont «acceptables» du point de vue de leur éthique professionnelle3. Les nouvelles techniques médicales suscitent donc des dilemmes éthiques sans précédent qui obligent les équipes hospitalières et les parents à s'interroger sur le bien-fondé et les éventuelles limites de ces soins.

\footnotetext{
${ }^{1}$ Voir R. Robert, E. Ferrand, «Limitation et arrêt des thérapeutiques en réanimation : expérience de dix centres », Réanim Urgences 1996; 5, p. 611-616. Et M. Dehan, «Grands prématurés : enjeux éthiques de la décision en néonatologie », in Fins de vie et pratiques soignantes, Espace éthique AP/HP, hiver 2000, p. 75-78.

${ }^{2}$ Parmi les affections les plus sévères que peuvent rencontrer les grands prématurés survivants, on compte la leucomalacie périventriculaire qui survient chez 5 à 15\% des prématurés selon l'âge gestationnel.

${ }^{3}$ Enquête EURONIC, in M. Cuttini et al. (2000), « End of life decisions », Lancet, 355, p. 2112-2118.
} 
Les actes réalisés consistent bien en une euthanasie, quelle que soit la procédure réellement employée et même si cette dénomination n'est jamais utilisée par les protagonistes. La situation néonatologique, et les arguments qu'elle convoque, diffèrent pourtant fondamentalement de la fin de vie. Ni le «droit» de choisir sa mort ni l'argument de l'autonomie de la volonté ou du consentement n'interviennent ici, le « patient » étant en outre privé de la capacité d'exprimer sa volonté ${ }^{1}$. Autrement dit, on juge pour lui.

La confrontation à ces situations cliniques donne lieu à une élaboration théorique et normative de principes. Celle-ci advient dans un contexte de divergences éthiques insolubles, où sont convoqués des jugements qui sont non seulement médicaux mais aussi moraux, éthiques et juridiques. La référence à des principes, à des normes et à des valeurs morales ne permet aucunement de résoudre a priori les questions éthiques qui se posent. Les dilemmes suscités sont d'autant plus aigus que se trouvent en présence des normes morales dont chacune a une légitimité restreinte.

En raison des conflits éthiques que nourrissent ces pratiques médicales et du fait de leur usage pourtant fréquent, il est indispensable qu'elles fassent l'objet d'une prise en compte institutionnelle. Pour le montrer, nous envisagerons, en premier lieu, les modalités d'élaboration des normes éthiques visant à encadrer les pratiques de non réanimation en France et aux Pays-Bas. Nous préciserons ensuite le cadre normatif dans lequel interviennent ces pratiques et analyserons les principes en référence auxquels elles se justifient. Enfin nous présenterons les linéaments d'une organisation institutionnelle des désaccords éthiques, irréductibles dans ce domaine.

\section{L'élaboration de principes à partir de situations cliniques.}

La confrontation à des situations cliniques difficiles a induit en France un processus d'élaboration théorique et normative dont l'Avis n65 («Réflexions éthiques autour de la réanimation néonatale ») du Comité Consultatif National d'Ethique (CCNE) témoigne. Il n'existe pas, en France, de cadre juridique qui limite les circonstances dans lesquelles les médecins seraient autorisés à arrêter la réanimation d'un nouveau-né. L'arrêt de la réanimation, même parfaitement motivé, entraînant volontairement la mort, constitue une transgression des lois juridiques. Cette transgression se trouve pourtant justifiée voire légitimée par le CCNE qui s'exprime en ces termes :

«Certes, il s'agirait [...] d'une transgression évidente de la loi qui interdit toute atteinte à la vie humaine. Mais peut-être faut-il, face à des drames pour lesquels il n'existe aucune solution satisfaisante, comprendre une telle transgression $»^{2}$.

La justification de pratiques juridiquement transgressives repose toutefois sur des arguments moraux et sur la référence à des "grands principes »- tels que le refus de l'acharnement thérapeutique - ainsi que sur la légitimité du fait de suivre une procédure conduisant à un choix éclairé et raisonnable. Cette référence procédurale est l'un des éléments décisifs évoqués aux Pays-Bas en faveur des arrêts médicalisés de vie. La légitimité de la décision s'enracine dans le fait de se soumettre à une procédure stricte (écartant les décisions arbitraires dans le domaine), à une "démarche transparente et progressive, menée en conscience, avec humanité, dans le souci de respecter au mieux les parents de l'enfant, seuls à

\footnotetext{
${ }^{1}$ La singularité et la spécificité de la situation néonatologique ne tient pas tant au fait que les personnes concernées sont privées de la possibilité de s'exprimer ou de formuler des jugements sur la qualité de vie qui pourra être la leur dans le futur que sur le fait que l'on pratique l'euthanasie sur des personnes qui n'ont jamais vécu.

${ }^{2}$ Comité Consultatif National d'Ethique pour les sciences de la vie et de la santé, Ethique et recherche biomédicale : rapport 2000, Paris, La Documentation française, 2003, p. 87.
} 
pouvoir prendre la mesure du poids de l'avenir qui s'ouvre devant eux », pour reprendre les termes de l'Avis cité du CCNE (p. 87).

La référence faite au cadre procédural ne peut certes intervenir qu'à partir du moment où l'on accepte de surseoir, dans certains cas au moins, au principe de préservation de la vie à tout prix. Cependant l'Avis du CCNE est intrinsèquement problématique. En premier lieu, il formule un principe qui tend à passer pour une loi. Il produit des effets de normativité chez les acteurs alors même que, d'un point de vue juridique, aucune exception, en la matière, n'est acceptable et que cette exception se voit légitimée hors de toute référence juridique ${ }^{1}$. De ce point de vue, le protocole de Groningen est plus satisfaisant.

Le protocole de Groningen, élaboré par plusieurs néonatologistes hollandais principalement E. Verhagen et $\mathrm{P}$. Sauer - à partir de leurs propres pratiques de réanimation, consiste en une procédure permettant de réguler et d'encadrer le " do not resuscitate order " $(\mathrm{DNR})^{2}$. Il est défendu par ses concepteurs au motif, notamment, qu'il offre un cadre juridique permettant d'éviter les abus.

\section{Justification procédurale de pratiques transgressives.}

\subsection{La procédure garantit-elle l'acceptabilité éthique des décisions ?}

Le principe de l'euthanasie étant accepté aux Pays-Bas - et juridiquement institué par la loi hollandaise -, sa mise en œuvre ne se justifie que pour autant qu'elle répond à des conditions précises et s'effectue selon une démarche codifiée ${ }^{3}$. Dans les deux cas portés en justice aux Pays-Bas, dans les années 1990, le procureur a retenu quatre critères fondamentaux de justification : (i) l'absence d'espoir, des souffrances insupportables, une qualité de vie future très médiocre pour l'enfant; (ii) le consentement des parents; (iii) l'avis d'un médecin indépendant et sa convergence d'opinion avec les médecins traitants ; (iv) la mise en œuvre d'une procédure conforme aux normes médicales acceptées.

Le protocole est mis en œuvre en référence à trois types de cas : (a) Les enfants qui n'ont aucune chance de survie. (b) Les enfants dont le pronostic est très mauvais, qui seront dépendants de soins intensifs et dont on anticipe qu'ils auront une vie et une qualité de vie médiocres. (c) Les enfants dont le diagnostic est mauvais et dont les parents et les médecins considèrent que la souffrance est insupportable ${ }^{4}$.

Cette distinction entre trois types de cas engage des jugements normatifs en matière de qualité de vie. En particulier le recours au principe du refus de l'acharnement thérapeutique est motivé par des jugements normatifs - i.e. pas seulement par des pronostics médicaux -

\footnotetext{
${ }^{1}$ De la même façon, le CCNE dans son Avis n ${ }^{\circ} 63$ ( «Fin de vie, arrêt de vie, euthanasie ») formulait une « exception d'euthanasie » pour les personnes en fin de vie qui a aussi été jugée inacceptable. Elle instituait une irresponsabilité pénale incompatible avec le droit pénal français. Elle pouvait donner lieu à des contentieux et des conflits davantage qu'à des apaisements et à des solutions. La nature de cet avis a été l'une des raisons pour lesquelles la loi Léonetti a été adoptée.

${ }^{2}$ A. A. E. Verhagen et P. J. Sauer, « The Groningen Protocol - Euthanasia in Severely Ill Newborns », The New England Journal of Medicine, vol. 352, Mars 10, 2005, ${ }^{\circ} 10$, p. 959-962. Le protocole de Groningen établit que les parents, l'équipe médicale et les travailleurs sociaux doivent tous reconnaître que les traitements sont devenus « futiles » (inutiles). Après quelques jours durant lesquels les parents prennent le temps de réfléchir à la décision et de dire adieu à leur enfant, ce dernier est tué soit avec de la morphine ou du midazolan. Un rapport est fait au procureur de la république sur l'acte d'arrêt de traitement.

${ }^{3}$ Le respect de la procédure et des étapes qui précèdent l'acte qui conduit à la mort est aussi un élément central de la Loi française $\mathrm{n}^{\circ} 2005-370$ du 22 avril 2005 relative aux droits des malades et à la fin de vie.

${ }^{4}$ On distingue la souffrance de la douleur, cette dernière se définissant à partir du fait qu'elle peut être soulagée par des antalgiques.
} 
quant à ce que sera la qualité de vie future de l'enfant. On considère que la survie de l'enfant ne justifie pas, à elle seule, les soins mais que le but de ces derniers est une «qualité de vie acceptable $»^{1}$. On admet et conclut qu'il est « de l'intérêt de l'enfant » de ne pas s'engager dans une intervention médicale - autrement dit qu'il vaut mieux pratiquer une euthanasie lorsque « les perspectives sont mauvaises ». Ainsi E. Verhagen et P. Sauer considèrent qu'une vie de souffrances, qui ne peuvent être soulagées, fait partie de ces cas extrêmes où lorsque la vie est volontairement interrompue, on ne peut parler de meurtre. Ici comme c'est le cas dans l'Avis du CCNE, la décision d'arrêt de vie (ou de ne pas maintenir la survie artificielle) est explicitement justifiée à partir des conséquences que subira l'enfant du fait de la décision médicale de poursuivre la réanimation mais également à partir du poids que constituera cette vie, certainement très lourdement handicapée, pour la famille de l'enfant ${ }^{2}$.

Plusieurs difficultés se présentent. Non seulement la décision de poursuite ou d'arrêt du traitement intervient toujours dans un contexte d'urgence et d'incertitude ${ }^{3}$, mais il n'existe surtout aucun consensus sur le bénéfice recherché et sur la hiérarchie des priorités devant guider les actes. Certains insistent sur la préservation de la vie de l'enfant, d'autres sur les handicaps engendrés, qui entraveront son épanouissement autonome et pèseront lourdement sur les liens familiaux. Or l'élaboration et la mise en œuvre de ces protocoles présupposent qu'il est possible de parvenir à un accord sur la priorité à conférer à tel principe plutôt qu'à tel autre. Précisément, les procédures placent en leur cœur le fait que les participants - équipe soignante et parents - parviennent à un " consensus ». Mais ce consensus suppose la mise en accord (et certainement la hiérarchisation) de principes qui ont chacun une légitimité restreinte et qui ont pu, dans un premier temps, entrer en conflit. La référence à des " grands principes éthiques » justifie, dans les recommandations du CCNE, la transgression admissible de la loi et du principe du respect absolu de la vie humaine mais ces "principes éthiques » peuvent-ils réellement être mis en accord ou hiérarchisés ?

\subsection{La référence à des « grands principes éthiques ».}

Parmi les grands principes éthiques convoqués, on compte le principe du respect de la vie, l'obligation de soins, le principe de bienfaisance ou principe de «non nuisance », le refus de l'acharnement thérapeutique ou de l' «obstination déraisonnable », «l'intérêt supérieur » de l'enfant, le principe de compassion. Le refus de l'acharnement thérapeutique, qui motive les décisions adoptées à l'égard de la seconde catégorie d'enfants, décrite par le protocole de Groningen, est admis par tous les néonatologistes hollandais et par la majorité des néonatologistes européens, qui considèrent que les soins intensifs ne sont pas une fin en soi.

La principale ligne de clivage éthique est celle qui sépare les partisans de la préservation de la vie de l'enfant, d'une part, et, d'autre part, ceux qui insistent sur les handicaps à venir qui limiteront l'épanouissement autonome de ce dernier et pèseront sur les liens familiaux. De la même façon, le principe du refus de l'«obstination déraisonnable » peut être défendu contre celui de l'obligation de soins. Le principe de «non nuisance», pour sa part, est intrinsèquement problématique. Le serment d'Hippocrate fait du précepte Primum non nocere l'un des fondements de la médecine, lequel consiste dans le respect de quatre impératifs : ne pas nuire, prévenir un effet nocif, supprimer un effet nocif, apporter un effet bénéfique. Or dans les situations évoquées, il est particulièrement délicat d'identifier les traitements et

\footnotetext{
${ }^{1}$ A. A. E. Verhagen et P. J. Sauer, « The Groningen Protocol - Euthanasia in Severely Ill Newborns ».

${ }^{2}$ CCNE, Avis $n^{\circ} 65$, p. 81 et p. 93 .

${ }^{3}$ Les situations les plus délicates sont celles où sont constatées des lésions cérébrales, susceptibles d'entraîner des séquelles graves et irréversibles qui obèrent fortement tout espoir de vie relationnelle de l'enfant mais restent compatibles avec la possibilité d'une survie.
} 
pratiques qui correspondent strictement à chacune de ces intentions. En particulier, il est difficile de parvenir à une certitude " raisonnable » que telle ou telle action sera bénéfique. Pour certains, l'impératif de « ne pas nuire » peut signifier éviter «l'apparition d'un handicap majeur qui remettrait en cause le principe de bienfaisance $»^{1}$. Ces conflits de normes conduisent à penser que l'on touche peut-être ici aux limites de l'élaboration normative dans la mesure où l'on s'efforce de hiérarchiser des principes qui sont individuellement fondamentaux mais incommensurables entre eux. Cependant, dans la pratique, s'organisent des arbitrages de normes qui débouchent sur des hiérarchisations de principes et des attributions de priorités ${ }^{2}$.

Dans la mesure où les arrêts de réanimation et les arrêts de vie conduisent à assumer le paradoxe d'une transgression de ce que l'on considère comme un principe intransgressible (i.e. le respect de la vie), on ne peut déclarer éthique, de manière a priori, une décision de fin de vie. Néanmoins la légitimité éthique de ces actes peut être, pour une part, éclairée et garantie par une réflexion sur les modalités de prise de décision et d'application de la décision.

\subsection{Discussion argumentative et délibération collective : des sources de légitimation.}

La référence aux "grands principes » éthiques advient dans le cadre d'une démarche argumentative et/ou d'une délibération collective, présidant à la décision de poursuivre ou d'interrompre des soins. Le cadre délibératif garantit le respect de ces principes éthiques et contribue à leur hiérarchisation dans chacun des cas pour lequel une délibération collective est initiée. Ainsi la réanimation néonatale et les décisions de NTBR offrent un exemple d'éthique de la discussion et de recherche du consensus dans le dialogue. La discussion joue le rôle de critère de validation et de légitimation de la décision.

Dans la plupart des pays dont la France, la décision d'arrêt de réanimation se fait le plus souvent de façon collective. Le protocole de Groningen et l'Avis n ${ }^{\circ} 65$ du CCNE insistent sur l'exigence que la décision soit prise de façon collégiale (i.e. que le médecin ne la prenne pas seul) et, aux Pays-Bas, qu'un consentement informé et réel de la part des parents soit obtenu. L'élaboration collective de la décision, dans ce cadre d'éthique procédurale, a pour vocation de fournir à l'enfant et à sa famille des garanties. La discussion et la délibération collective visent à identifier le «raisonnable » et le "déraisonnable », en l'occurrence l'obstination déraisonnable, dans un contexte où les limites de l'acharnement thérapeutique ne peuvent malheureusement pas être définies et identifiées à partir d'un ou de plusieurs critères absolus et incontestables mais seulement à partir d'un faisceau d'indicateurs de différents ordres.

Ce cadre délibératif doit permettre d'écarter la prédominance de logiques individuelles, où chacun prendrait position, spontanément, en fonction de ses appartenances culturelles, de ses convictions, de son état psychologique et de ses propres capacités à faire face aux situations. Les convictions personnelles ou collectives méritent d'être reconnues mais elles doivent être, corrélativement, mises à distance de façon critique. La délibération doit permettre aux parties prenantes d'éliminer de leurs raisons d'agir des motifs inconscients ou conscients qui seraient

\footnotetext{
${ }^{1}$ CCNE, Avis n ${ }^{\circ} 65$, p. 79.

2 Ainsi les médecins hollandais, qui ont procédé aux 22 euthanasies ayant fait l'objet d'un rapport au cours des sept dernières années, pour arrêter la vie des nouveaux nés, ont dans $100 \%$ des cas, pris en compte la très faible qualité de vie en termes d'absence de souffrances, handicap fonctionnel, douleur, absence d'espoir, pronostic pessimiste ainsi que l'absence anticipée d'autonomie. Dans $82 \%$ des cas, il ont pris en compte l'incapacité anticipée à communiquer, dans $77 \%$ des cas, la dépendance à l'égard de la structure hospitalière et dans $59 \%$ des cas l'espérance de vie.
} 
« autres que l'intention droite d'éviter l'obstination déraisonnable pour tel patient singulier $»^{1}$. Cette exigence incarne le principe de publicité kantien selon lequel : « ce dont on ne peut pas dire qu'on le fait, on ne doit pas le faire $»^{2}$. Ainsi les motifs auxquels les individus font référence, pour justifier et argumenter leurs positions sur une décision d'euthanasie, doivent pouvoir être universalisés ${ }^{3}$. Les partenaires de la décision doivent pouvoir répondre affirmativement à la question : peut-on penser que tout le monde ferait ce que l'on décide de faire, et peut-on vouloir que tout le monde, dans la même situation, fasse ce que l'on décide de faire ? Si l'intention et le motif permettent de répondre affirmativement à ces questions, alors ils sont moralement acceptables et la délibération collective consiste dans la mise en œuvre de l'exigence kantienne d'universalisation de la maxime.

La légitimité des décisions dépend certes du respect d'un cadre formel mais il importe de ne pas s'enfermer dans une logique procédurale : la pertinence des décisions et des actes ainsi que leur légitimité ne sont pas conférées a priori par la procédure, fût-elle reconnue par tous. Lorsque la délibération collective ne parvient pas à un consensus, celui-là peut être recherché à travers une discussion argumentative, convoquant des éléments objectifs et des arguments moraux en vue de parvenir à une décision ${ }^{4}$. Le dissensus des participants recouvre en effet souvent des divergences éthiques sous-jacentes. La validité morale du résultat auquel conduit l'argumentation se voit garantie par le respect des conditions du dialogue, établies par l'éthique de la discussion ${ }^{5}$. Celle-ci - conformément à l'interprétation habermassienne ${ }^{6}-$ permet de faire émerger une rationalité morale intersubjective et contribue, par conséquent, à déterminer ce qui est souhaitable, préférable (i.e. bien).

Le respect des conditions de l'éthique de la discussion, en l'occurrence de la communication et du dialogue, signifie notamment que le charisme personnel et la position hiérarchique doivent être régulés par une distribution équitable de la parole et une incitation faite à chacun de s'exprimer. Un climat de confiance doit autoriser chaque participant à exprimer son assentiment ou à émettre des réserves. Lorsqu'un avis différent est formulé, une attention particulière doit lui être portée afin de préciser les éléments qui en sont à l'origine. Il faut être vigilant au fait que (a) toutes les personnes concernées aient une même chance de contribuer à la constitution d'un compromis équitable ; que (b) certaines règles pragmatiques de l'argumentation sont respectées comme le fait que chaque personne concernée ait eu toutes les possibilités de donner son adhésion de plein gré et qu'aucun des participants n'impose aux autres l'issue des dilemmes envisagés ${ }^{7}$, comme ce pourrait être le cas dans des rapports hiérarchiques entre membres des équipes soignantes, chefs de service et infirmières, entre la hiérarchie du service de néonatologie et les parents des enfants concernés ${ }^{8}$, ou bien dans les débats publics où les positions des partenaires sont asymétriques, en particulier s'agissant de la défense de convictions idéologiques minoritaires, dans une société majoritairement libérale.

\footnotetext{
${ }^{1}$ S. Grobuis, F. Nicolas, S. Rameix et al., « Bases de réflexion pour la limitation et l'arrêt des traitements en réanimation chez l'adulte », Réanim Urgences 2000, 9, Article spécial, p. 19.

${ }^{2}$ Voir E. Kant, Projet de paix perpétuelle, in Euvres complètes, Paris, Gallimard, 1985, tome II, p. 377-378 et p. 382 .

${ }^{3}$ Selon une distinction entre les intentions et les mobiles : le mobile étant ce qui nous pousse à agir, qu'on le sache ou non. L'intention désigne en revanche les raisons que nous avouons pour expliquer notre agir (voir J.-F. Malherbe, Homicide et compassion. L'euthanasie en éthique clinique, Montréal, Médiaspaul, 1996, p. 31-32).

${ }^{4}$ Afin de ne pas fonder la décision exclusivement ou de façon prédominante sur des critères médicaux et techniques.

${ }^{5}$ Car la collégialité ne suffit pas à garantir le caractère éthique de la décision.

${ }^{6}$ Voir J. Habermas, Morale et communication, 1986, Paris, Cerf.

${ }^{7}$ Nous empruntons certaines des suggestions ici faites à H. Pourtois, « Délibération, participation et sens du désaccord », Ethique publique, vol. 7/n¹ (2005), p. 145-154.

${ }^{8}$ Ce type d'exigence conduit le GFRUP à recommander que «les services se dotent d'un espace où le questionnement du personnel soignant puisse être exprimé de façon régulière » (P. Hubert et al., « Limitations et arrêts de traitements actifs en réanimation pédiatrique : recommandations du GFRUP », p. 1504).
} 
Il est décisif que le compromis auquel on parvient, à quel que niveau que ce soit, ne dépende pas exclusivement des positions initiales de force ou de faiblesse des partenaires de la discussion.

Cette éthique de la communication et du dialogue suppose que lorsque survient un désaccord profond de l'un des participants, à partir d'arguments sérieux et éprouvés, la prise de décision doit être différée et conduire éventuellement à ne pas pratiquer l'arrêt de vie. Ainsi on a vu émerger, aux Etats-Unis à la fin des années 1990, un principe de partage de la décision, permettant que toute personne impliquée dans celle-ci, si elle a le sentiment qu'une autre partie n'a pas comme principal objectif l'intérêt supérieur de l'enfant a une obligation d'objection.

Il peut enfin, au terme de la discussion, subsister des divergences qui sont sans rapport avec la validité de la décision ni avec le respect des conditions dans lesquelles la discussion s'est déroulée. Certains acteurs peuvent éprouver un sentiment de transgression suscité aussi bien par des convictions éthiques propres que par des expériences personnelles antérieures. Le cadre délibératif impose de tenir compte des limites de ce qui est acceptable pour chacun ${ }^{1}$. Le dissensus doit être accepté, toléré et reconnu comme l'une des possibilités intrinsèques des procédures de dialogue identifiées par ailleurs comme étant l'un des garants de la valeur éthique des décisions finalement prises.

Le désaccord peut aussi bien survenir dans les équipes médicales elles-mêmes que dans le débat public aussi peu ouvert soit-il en France sur ces questions. Il sera d'autant plus récurrent que l'expression équitable des convictions de chacun sera respectée. Ces situations de désaccords persistants appellent des dispositions institutionnelles tenant compte des convictions éthiques divergentes en présence. Cette institutionnalisation est d'autant plus justifiée qu'existe aujourd'hui en France un décalage problématique entre les dispositions juridiques existantes et la transgression rendue acceptable par les recommandations du CCNE au milieu d'un complet vide juridique en matière de réanimation néonatale. Un exemple de cette institutionnalisation a été récemment fourni par la loi Léonetti du 22 avril 2005, concernant le droit des malades et la fin de vie, adoptée à la suite des débats suscités par le cas Vincent Humbert.

\section{L'institutionnalisation des désaccords.}

Face à ce décalage et à ces désaccords, certains, comme Marc Grassin, considèrent qu'il est préférable d'accepter que les arrêts de réanimation ne soient pas d'abord l'enjeu d'une régulation dans la mesure où ils nous placent dans une situation limite et parce que nous ne saurions penser les décisions de fin de vie comme une pratique sociale comme une autre ${ }^{2}$. Il nous semble pourtant préférable de faire droit à une législation inspirée du protocole de Groningen associé à d'autres mesures, que nous allons évoquer, plutôt que de demeurer dans le cadre normatif proposé par l'Avis n ${ }^{\circ} 65$ du $\mathrm{CCNE}^{3}$.

\subsection{L'organisation du désaccord éthique : du consensus au compromis.}

\footnotetext{
${ }^{1}$ Voir J. Rawls, Libéralisme politique, Paris, PUF, 2001, p. 91.

${ }^{2}$ M. Grassin, Les arrêts de réanimation et les arrêts de vie en néonatologie [...], Thèse, 1999 ; conclusion.

${ }^{3}$ Pour une critique de l'argument de la «pente fatale», voir J.-Y. Goffi, Penser l'euthanasie, Paris, PUF, 2004, p. 29-35.
} 
Les dilemmes qui se posent en réanimation néonatale suscitent un désaccord moral profond à propos duquel l'équilibre entre les libertés de base (le droit à la vie, le droit aux soins, etc.) s'appuie sur des valeurs en conflit, dont l'interprétation les rend difficilement conciliables. Les parties en présence, le plus souvent, s'accordent à un certain niveau de généralité sur ces principes fondamentaux mais non sur les détails. Le débat public met en présence des valeurs morales qui n'ont pas moins de poids que d'autres types de valeurs ou de considérations, utilitaristes ou pragmatiques par exemple. Les arguments qui se font face sont fondés sur de bonnes raisons, compréhensibles par tous. Aucun consensus moral ni certainement aucun consensus politique ne résultera des discussions comme les faits, concernant d'autres situations d'euthanasie, sont là pour le prouver. Qui plus est, il n'est pas certain qu'il faille vouloir systématiquement régler les conflits éthiques définitivement sous la forme d'un consensus moral. Non seulement il est douteux que l'argumentation proprement politique parvienne à faire disparaître les conflits moraux et les conflits de valeurs, mais il est peut-être aussi illégitime de tenir pour acquis qu'elle y parvienne. Dans cette mesure, il est préférable d'organiser un modus vivendi responsable plutôt que de vouloir le dépasser comme le souhaite Rawls - dans un consensus par recoupement ${ }^{1}$.

L'organisation structurée du désaccord, sur ces questions, suppose de prendre en considération les convictions éthiques des parties en présence sans privilégier l'expression de certaines valeurs morales sur d'autres, dans le débat public comme dans la pratique. Dans un contexte libéral, le souhait, motivé par le principe de non nuisance, de ne pas être responsable de la vie d'enfants très lourdement handicapés ${ }^{2}$ ne peut être d'emblée disqualifié. Les conceptions du bien en présence, nourrissant une éthique du respect de la vie ou une éthique de la qualité de vie, fournissent toutes de " bonnes raisons » de préférer tel ou tel type de décision.

Les dilemmes éthiques étant ici irréductibles, on doit pouvoir admettre que des personnes puissent ne pas supporter, non pas simplement pour des raisons pratiques ou pragmatiques, d'avoir un enfant lourdement handicapé ni l'imposer à un autre que soi - ce qui est le cas lorsque «l'acharnement thérapeutique » est poursuivi jusqu'au dernier degré. Les médecins anesthésistes réanimateurs, dans certains cas, sont en position d'imposer à des parents des orientations de vie problématiques, y compris à des parents privilégiant une éthique de la qualité de vie sur une éthique du respect inconditionné de la vie et qui décideraient, s'ils le pouvaient, l'arrêt de la réanimation. Incontestablement l'enfant, quelles que soient les pathologies dont il peut être affecté, doit être protégé et tel est le devoir des institutions. Toute la difficulté est cependant ici de concevoir la façon dont divers types de raisons - également légitimes moralement - peuvent intervenir dans la préparation des décisions. Les convictions éthiques des parents demandent à être entendues tout de même qu'il est indispensable de tenir compte des limites de ce qu'autrui peut accepter et vivre ${ }^{3}$.

\footnotetext{
${ }^{1}$ Voir C. Arnsperger \& E. Picavet, « More than modus vivendi, less than overlapping consensus : towards a political theory of social compromise », Information sur les sciences sociales/Social Science Information, 43 (2), Juin 2004, p. 167-204.

${ }^{2}$ Evoquons simplement le cas du petit Olivier, né à 25 semaines de grossesse au Québec, le 21 octobre 1996. Il présente une paralysie cérébrale des quatre membres mais avec une atteinte moindre aux mains. Il est aveugle et a des problèmes pulmonaires sévères ainsi que de nombreuses allergies. Il était affecté notamment d'une leucomalacie périventriculaire, d'une importante dysplasie bronchopulmonaire, d'une rétinopathie du prématuré très sévère (de grade IV). Il présentait également à la naissance une hernie inguinale et une atrésie (une malformation) de l'œsophage. Olivier a été hospitalisé des dizaines de fois dont au moins à 16 reprises pour des opérations concernant ses problèmes pulmonaires, son atrésie de l'œsophage et ses yeux, pour le reflux, pour sa gastrotomie ou parce qu'il s'était étouffé. En neuf ans, ce n'est qu'au cours des mois de mars 2003 à mars 2004 , qu'il n'a pas été hospitalisé.

${ }^{3} \mathrm{C}$ 'est ce que suggère le CCNE en soulignant que « le dialogue tiendra compte des forces et des faiblesses des parents qui auront à assumer la charge de l'enfant après sa sortie des soins intensifs » (Avis nº6, p. 10).
} 
Privilégier le dialogue ou le «compromis » au détriment du consensus doit permettre d'atténuer l'inégalité de traitement des individus, dont les valeurs personnelles profondes pourraient ne pas être prises en compte. Cette option est d'autant plus pertinente qu'elle incarne un principe d'impartialité à l'égard des intérêts et des valeurs des parties en présence. La reconnaissance de l'irréductibilité des conflits de valeurs comme des critères de jugement exprime une prise en compte du pluralisme dans sa radicalité.

Notre propos est de suggérer qu'une expression institutionnelle réelle de ce dernier peut être trouvée y compris sur les questions évoquées. Dans cette perspective, il ne s'agit pas seulement de reconnaître le pluralisme des conceptions individuelles du bien - c'est-à-dire une forme de pluralisme " affaiblie " - mais bien de faire une place à un pluralisme radical, induit par l'incompatibilité des conceptions morales des uns et des autres, par leur incommensurabilité et la non-comparabilité des valeurs.

L'organisation structurée des désaccords et de leur expression implique que demeure ouverte, dans le débat public, la possibilité de remises en cause ultérieures des résolutions adoptées. Elle reflète la conscience des virtualités positives d'un désaccord conscient et entretenu comme du débat public contradictoire. On admet alors qu'il peut y avoir des aspects positifs au maintien de positions irréconciliables permettant par exemple de corriger l'inégalité des individus, dans ces cadres délibératifs et face aux risques de frustration de leurs valeurs personnelles les plus profondes, mais également en matière de transparence des pratiques ou d'expression de l'objection de conscience par les agents. Cette institutionnalisation du désaccord appelle une organisation spécifique de la confrontation des conceptions morales dans la sphère publique comme dans les organisations sociales. Comment pour la question qui nous occupe est-il possible d'organiser la coexistence et l'expression séparée des convictions, dans les institutions, d'une manière qui ne mène pas au conflit et permette aux agents de coopérer?

\section{2 « Organiser » le désaccord éthique.}

\subsubsection{Comment respecter institutionnellement le pluralisme éthique?}

L'organisation du désaccord présente plusieurs dimensions. Quoique d'un point de vue instrumental et strictement politique, il est requis que les citoyens admettent que leurs différends sur l'interprétation des droits généraux proclamés sont secondaires, on ne peut justifier l'existence d'une exigence morale imposant que les citoyens abandonnent leurs désaccords sur l'interprétation des principes fondamentaux évoqués et remanient leurs valeurs personnelles dans la direction ainsi ouverte. La manière dont des exigences morales précises sont concrétisées dans les institutions est décisive. Des mesures respectant les valeurs lésées doivent donc être recherchées.

En particulier, on peut considérer que pour les raisons évoquées, il est préférable de retenir certaines normes fondamentales à titre provisoire. De la même façon, il serait judicieux de suspendre l'application de normes controversées, ayant des enjeux touchant à la vie et à la mort, tout aussi bien que de renoncer à consacrer sous forme de droits absolus des actes qui suscitent la mésentente ${ }^{1}$ et dont les questions relatives à l'euthanasie offrent un cas paradigmatique. De la sorte, la possibilité de remises en cause ultérieures des normes contestées demeurerait. Le cas échéant, ceux dont le point de vue n'est pas reflété dans la loi se trouvent dans une situation où des choses légales sont injustes à leurs yeux et des choses

\footnotetext{
${ }^{1}$ Voir E. Picavet, « Biens communs, valeurs privées et fragilité de l'Etat de droit », in Ethique Publique, vol. 6, n¹, 2004 (Québec), p. 98.
} 
illégales justes. De la même façon et s'agissant de conflits éthiques, engageant la vie et la mort, on pourrait ne rien institutionnaliser qui soit inacceptable pour aucune des parties en présence. Ces dispositions permettraient de préserver des chances équitables de contestation des droits controversés, des principes publiquement reconnus ou des pratiques médicales courantes, tout en tenant compte des frustrations et de l'indignation éthique de ceux qui ont dû s'incliner'. Le souci de privilégier le compromis sur le consensus doit enfin être soutenu par la création de lieux d'expression du dissentiment sur les questions éthiques, tels que les comités publics d'éthique dont les modalités de fonctionnement pourraient être ajustées à la persistance des désaccords éthiques.

\subsubsection{Permettre l'expression de convictions éthiques divergentes au sein des pratiques.}

Concernant les questions d'éthique médicale, les oppositions et divisions ont souvent une base éthique. Elles révèlent l'existence de communautés éthiques différenciées. Il est donc indispensable de mener une réflexion précise sur les modalités d'application des décisions prises - fussent-elles éthiquement fondées - car ces modalités peuvent devenir une source de conflit (et donc de souffrance) avec les parents ou au sein de l'équipe soignante, et de se demander : qui procède aux actes ? quand ? En présence ou non des parents ${ }^{2}$ ? Comment ces derniers sont-ils ensuite pris en charge? Leur laisse-t-on un temps de réflexion entre la décision et son exécution?, etc. On constate que dans les pratiques des médecins américains (et de certains réanimateurs) étudiés par Meier $^{3}$ sans qu'il s'agisse spécifiquement de la néonatalogie, seuls $43 \%$ des praticiens décidant d'une injection létale la pratiquent euxmêmes. Dans les autres cas, l'infirmière $(57 \%)$ ou un collègue $(32 \%)$ effectuent l'acte, ou bien une prescription simple d'augmenter les doses de médicaments type opioïdes ou barbituriques est effectués (11\%).

Dans la mesure où certains acteurs peuvent alors éprouver un sentiment de transgression suscité aussi bien par des convictions éthiques spécifiques que par des expériences personnelles passées, il importe de tenir compte de ce sentiment et de ne pas demander aux personnes qui l'éprouvent (médecins ou infirmières) d'exécuter les gestes conduisant à un arrêt de la vie. Il est indispensable d'organiser le "vivre ensemble » et le "travailler ensemble » de personnes qui ne partagent pas les mêmes convictions éthiques. Le cas échéant, les agents non seulement éprouvent une réelle difficulté à vivre et à travailler ensemble, mais le fonctionnement des équipes médicales peut également être perturbé par ces divergences éthiques.

L'institutionnalisation du désaccord, dans le domaine de la réanimation y compris néonatale, peut s'inspirer de l'organisation du service national en France, tel qu'il existait avant la suppression du service militaire obligatoire. Il permettait une différenciation des rôles civils et militaires et offrait une reconnaissance légale de l'objection de conscience. Le respect des convictions individuelles trouve déjà, dans l'exercice médical, son expression pratique dans la clause de conscience. De même, les équipes de réanimation pourraient être constituées

\footnotetext{
${ }^{1}$ Voir E. Picavet, «L'inégalité face aux principes publics », Humanistyka i przyrodoznawstwo, Olsztyn, 12 (2006) : 25-44, p. 39.

${ }^{2}$ Il est par exemple recommandé de toujours offrir aux parents la possibilité d'être présents au moment du dernier souffle de leur enfant, sans les culpabiliser s'ils ne peuvent ou ne veulent pas être présents (Direction des hôpitaux. Circulaire DH/EOS/98 n68 du 23 novembre 1998 relative au régime de visites des enfants hospitalisés en pédiatrie).

${ }^{3}$ D.E. Meier, C.A. Emmons, S. Wallenstein, T. Quill, R.S. Morissson, C.K. Cassel, « A national survey of physician assisted suicide and euthanasia in the united states ", New England Journal Medecine, 1998 ; 338 : 1193-201. Sur l'implication des infirmières dans les actes d'arrêt, voir A. Paillet, Sauver la vie, donner la mort, Paris, La Dispute/SNEDIT, 2007, p. 233-236.
} 
sur la base d'affinités éthiques partagées. Il est ainsi manifestement possible d'instituer des « parois » éthiques dans les institutions.

Concernant les parents, on peut imaginer qu'au début de la grossesse, ils puissent s'adresser à des services hospitaliers dont ils partageraient des convictions éthiques fondamentales engageant la vie et la mort. Ces dispositions soulèveront en France les critiques des partisans de la neutralité des institutions publiques. Notre propos n'est pas de justifier l'instauration d'options éthiques sur quelle que question que ce soit mais spécifiquement pour celles qui convoquent des enjeux vitaux et à laquelle échappe, par exemple, le souhait d'être soigné par une personne de son sexe. Les adversaires de toute forme d'euthanasie doivent pouvoir voir leurs convictions respectées d'autant qu'ils assumeront les conséquences de la survie d'un enfant lourdement handicapé si le cas se présente. Il est également aisé de faire droit au souhait des parents de ne pas être informés de la possibilité d'arrêter la réanimation ou d'arrêter la vie, dans une situation de réanimation où le pronostic vital de leur enfant est pessimiste en leur laissant la possibilité d'exprimer ce souhait avant de se trouver confrontés à cette situation ${ }^{1}$.

Ainsi on pourrait envisager, sur le modèle des solutions trouvées pour les situations de fin de vie, que soit distribué, lors des examens de suivi de grossesse, un questionnaire où les parents indiqueraient par avance leurs choix - toujours réversibles - dans le domaine. Ils indiqueraient par exemple s'ils souhaitent ou non être informés des risques et des possibilités d'arrêt thérapeutique, en cas de déficience de plusieurs fonctions vitales, nécessitant une suppléance technico-médicale, et de pronostic très péjoratif pour leur enfant, ce qui protègerait les parents les plus attachés au principe de la sacralité de la vie. En référence à la loi $n^{\circ}$ 99-477 du 9 juin 1999 visant à garantir le droit d'accès aux soins palliatifs, qui stipule qu'une offre de soutien doit être proposée aux familles au moment du décès ${ }^{2}$, le Groupe Francophone de Réanimation et Urgences Pédiatriques (GFRUG) suggère qu'elle prenne la forme d'un livret contenant les coordonnées des médecins et psychologues du service qu'ils peuvent contacter, des adresses de groupes de soutien pour parents endeuillés et éventuellement des références bibliographiques sur le deuil ${ }^{3}$. Ce livret pourrait évoquer les différents niveaux d'implication que les parents souhaiteraient avoir si la situation d'un arrêt possible de la vie se présentait à eux : (a) échanger des informations avec le médecin référent sans vouloir prendre de décision, (b) être informés et impliqués dans la prise de décision ${ }^{4},(\mathrm{c})$ ne pas recevoir d'informations trop précises. De la sorte, serait exprimé le souhait des parents à l'égard de l'intensité de leur engagement thérapeutique, en cas de naissance très prématurée et de la conduite à tenir en fonction du pronostic concernant la qualité de la vie future de leur enfant. Ce type de dispositif éviterait que la place des parents dans le partage éventuel de la décision ne se dessine de façon arbitraire ou systématique. Le médecin référent pourrait ainsi tenir compte des informations recueillies lors des questionnaires ou des entretiens préalables.

L'institutionnalisation et l'organisation du désaccord doivent être doublées d'une réflexion sur les conditions d'une culture du désaccord. Ainsi le respect d'une éthique de la communication et du dialogue lors des discussions comme le principe de la collégialité -

\footnotetext{
${ }^{1}$ Rappelons que l'avantage de la situation française est que la décision d'arrêt des soins vitaux, en dernière instance, revient aux médecins ce qui contourne la culpabilité que portent les parents, si la décision, comme c'est le cas aux Etats-Unis, leur revenait.

${ }^{2}$ Journal Officiel de la République Française, 1999 ; 10 : 8487-9.

${ }^{3}$ P. Hubert et al., « Limitations et arrêts de traitements actifs en réanimation pédiatrique : recommandations du GFRUP », p. 1507.

${ }^{4}$ Ce qui aurait pour conséquence que si les parents veulent partager la décision, le médecin référent doit savoir s'effacer pour les laisser prendre de l'autonomie et délivrer l'information de telle manière qu'ils puissent la recevoir comme une question. Si les parents ne souhaitent pas ou ne peuvent pas assumer cette décision, il faut leur permettre de se retrancher derrière la décision médicale, dont le médecin référent endossera la responsabilité.
} 
exercice nouveau dans les dispositifs actuels de soins - doivent faire l'objet d'un enseignement dans le cours des études médicales et paramédicales, tout de même que doivent être enseignées les qualités et compétences requises pour participer au processus de décision. Ces enseignements contribueront à rendre également les personnes sensibles à l'incidence émotionnelle que représente, pour chacun, la participation à une décision qui met en jeu la vie d'un enfant.

\section{Conclusion}

Comme nous l'avons montré, les pratiques de réanimation néonatale suscitent des dilemmes éthiques majeurs pour lesquels aucune solution a priori ne peut être trouvée et qui nourrissent des dissensions irréductibles. Cette situation justifie une institutionnalisation des désaccords éthiques respectant, dans la mesure du possible, les positions des défenseurs inconditionnels de la vie et le souci, partagé par d'autres, de la qualité de vie dont bénéficieront les enfants réanimés.

Les dispositifs de «séparation éthique» précédemment évoqués permettraient aux individus de coopérer, au sein des institutions, tout en ayant la possibilité de marquer leur différence et leur refus de certaines pratiques. L'avantage de tels dispositifs de « séparation éthique » serait d'assurer aux individus des garanties minimales et de les préserver contre les effets néfastes, dans leur propre vie, du triomphe de conceptions éthiques qui ne sont pas les leurs. En effet, la « victoire » de certaines valeurs sur d'autres impose un préjudice réel à une partie des citoyens, qui peut avoir des conséquences fâcheuses dans le déploiement de l'existence ordinaire comme sur leur lieu de travail. Sans défendre un «communautarisme éthique ", nous souhaitons souligner l'importance de prendre en considération les torts subis par les individus du fait de divergences éthiques et de la consécration sélective de certains principes, dans des sociétés libérales et technologiquement avancées.

L'institutionnalisation du désaccord - de ce fait, organisé -, assortie d'options individuelles ouvertes au choix comme celles que nous avons proposées, peut être une réponse satisfaisante au pluralisme éthique et à l'expression des convictions profondes des individus, en particulier lorsque des questions vitales sont en jeu. Elle paraît en outre préférable à un régime permanent de confusion sur les enjeux, de mésentente et de proclamation de principes autour desquels l'unanimité devrait se faire mais, dans les faits, ne se produit pas. Cette perspective, qui privilégie le compromis plutôt que le consensus, paraît d'autant plus désirable qu'elle serait le moyen de préserver des conditions équitables pour la concurrence entre des conceptions éthiques rivales au sein de la société et offrirait une solution à des pratiques largement répandues quoiqu'illégales et choquantes pour une partie de nos concitoyens.

\section{Références}

Verhagen A. A. E. et Sauer P. J., 2005, «The Groningen Protocol - Euthanasia in Severely Ill Newborns », The New England Journal of Medicine, vol. 352, Mars 10, $\mathrm{n}^{\circ} 10$, 959-962.

Comité consultatif national d'éthique pour les sciences de la vie et de la santé, Ethique et recherche biomédicale : rapport 2000, Paris, La Documentation française, 2003.

R. Robert, E. Ferrand, «Limitation et arrêt des thérapeutiques en réanimation : expérience de dix centres », Réanim Urgences 1996 ; 5, p. 611-616. 
M. Dehan, « Grands prématurés : enjeux éthiques de la décision en néonatologie », in Fins de vie et pratiques soignantes, Espace éthique AP/HP, hiver 2000, p. 75-78.

Enquête EURONIC, in M. Cuttini et al. (2000), «End of life decisions », Lancet, 355, p. 2112-2118.

S. Grobuis, F. Nicolas, S. Rameix et al., « Bases de réflexion pour la limitation et l'arrêt des traitements en réanimation chez l'adulte », Réanim Urgences 2000, 9, Article spécial.

E. Kant, Projet de paix perpétuelle, in Euvres complètes, Paris, Gallimard, 1985, tome II.

J.-F. Malherbe, Homicide et compassion. L'euthanasie en éthique clinique, Montréal, Médiaspaul, 1996.

H. Pourtois, "Délibération, participation et sens du désaccord », Ethique publique, vol. 7/n¹ (2005), p. 145-154.

M. Grassin, Les arrêts de réanimation et les arrêts de vie en néonatologie [...], Thèse, 1999.

Rawls J., 1993, tr. fr. 2001, Libéralisme politique, Paris, PUF.

J.-Y. Goffi, Penser l'euthanasie, Paris, PUF, 2004.

C. Arnsperger \& E. Picavet, « More than modus vivendi, less than overlapping consensus : towards a political theory of social compromise », Information sur les sciences sociales/Social Science Information, 43 (2), Juin 2004, p. 167-204.

E. Picavet, «Biens communs, valeurs privées et fragilité de l'Etat de droit », in Ethique Publique, vol. 6, $\mathrm{n}^{\circ} 1,2004$ (Québec).

E. Picavet, «L'inégalité face aux principes publics », Humanistyka i przyrodoznawstwo, Olsztyn, 12 (2006) : 25-44.

D.E. Meier, C.A. Emmons, S. Wallenstein, T. Quill, R.S. Morissson, C.K. Cassel, «A national survey of physician assisted suicide and euthanasia in the united states ", New England Journal Medecine, 1998 ; 338 : 1193-201.

A. Paillet, Sauver la vie, donner la mort, Paris, La Dispute/SNEDIT, 2007.

P. Hubert et al., «Limitations et arrêts de traitements actifs en réanimation pédiatrique : recommandations du GFRUP ». 